\title{
BMJ Open Lifestyle, genomic types and non- communicable diseases in Korea: a protocol for the Korean Medicine Daejeon Citizen Cohort study (KDCC)
}

\author{
Younghwa Baek (D) , ${ }^{1}$ Bok-Nam Seo, ${ }^{1}$ Kyoungsik Jeong, ${ }^{1}$ Horyong Yoo, ${ }^{2}$ \\ Siwoo Lee ${ }^{1}$
}

To cite: Baek Y, Seo B-N, Jeong $\mathrm{K}$, et al. Lifestyle, genomic types and noncommunicable diseases in Korea: a protocol for the Korean Medicine Daejeon Citizen Cohort study (KDCC). BMJ Open 2020;10:e034499. doi:10.1136/ bmjopen-2019-034499

- Prepublication history for this paper is available online. To view these files, please visit the journal online (http://dx.doi. org/10.1136/bmjopen-2019034499).

Received 24 September 2019 Revised 13 March 2020 Accepted 26 March 2020

Check for updates

(c) Author(s) (or their employer(s)) 2020. Re-use permitted under CC BY-NC. No commercial re-use. See rights and permissions. Published by BMJ.

${ }^{1}$ Future Medicine Division, Korea Institute of Oriental Medicine, Daejeon, Korea (the Republic of) ${ }^{2}$ Clinical Trial Center, Dunsan Korean Medicine Hospital of Daejeon University, Daejeon, Korea (the Republic of)

Correspondence to Dr Siwoo Lee; bfree@kiom.re.kr and Dr Horyong Yoo;

horyong.y00@gmail.com

\section{ABSTRACT}

Introduction Non-communicable diseases (NCDs) are the leading cause of death worldwide, including in Korea; thus, customised techniques for chronic disease management for the prevention, early diagnosis and treatment of NCDs are crucial. The Korean Medicine Daejeon Citizen Cohort (KDCC) study has been tasked with developing precise health promotion model for preventing and managing chronic diseases that considers individual traits, lifestyle factors and clinical data based on traditional Korean medicine.

Methods and analysis The KDCC study is a prospective cohort study of the community-based population in Korea. This study will sample 2000 adults aged $30-55$ years in Daejeon area using stratified cluster sampling. The baseline survey began in 2017 and was completed in 2019, and follow-up surveys will be conducted three times every 2 years until 2025. In follow-ups, factors related to cardiovascular disease, metabolic syndrome and relevant diseases, as well as respondents' health status information, will be examined via questionnaire surveys and health examinations.

Discussion The KDCC study will investigate the effects of an interaction of Korean medicine type with genome based, lifestyle and various clinical information on chronic diseases and individuals' health status using longitudinal epidemiological data. These findings are expected to inform the development of tailored health promotion programmes based on precision Korean medicine. Trial registration number KCT0004297.

\section{INTRODUCTION}

Non-communicable diseases (NCDs), which include cardiovascular disease (CVD), diabetes, chronic respiratory diseases and cancer, are the leading cause of death worldwide, accounting for about $70 \%$ of all deaths. Although national numbers vary according to the economy of each country, premature death caused by NCDs is consistently on the rise and has increased from 31.6 million in 2000 to 40.5 million in $2016^{2}$; further, premature death caused by CVD is high-at about $31 \%$ of all global deaths. ${ }^{34}$ Prevention
Strengths and limitations of this study

- The Korean Medicine Daejeon Citizen Cohort (KDCC) study is the first prospective cohort study to assess the causal relationships among Korean medicine types, lifestyle factors and chronic diseases.

- This study will present crucial results to clarify the biological features of Korean medicine types.

- Detailed clinical data will be collected, biological resources will be used and enhanced genomic analysis techniques will be employed to inform the clinical applicability of Korean medicine.

- KDCC longitudinal data will be used to develop a health promotion model for the prevention of chronic diseases based on Korean medicine types.

- Limitations of this study include the possibility of selection bias - owing to voluntary participation-and ungeneralisable findings - owing to participants being recruited from one region.

through lifestyle adjustment and control of risk factors is the key for NCDs, and diverse studies are underway to develop customised chronic disease management technologies, such as programmes for early diagnosis and treatment. ${ }^{56}$

In recent years, there have been attemptsprimarily centred on precision medicineto develop customised disease management by thoroughly examining individual traits and various external factors based on developments of genomic technology. ${ }^{7}$ Research concerning precision medicine is focused on cancer in the near term; however, the longterm aim is to develop knowledge applicable to a wide range of health issues and diseases, including chronic diseases, such as diabetes mellitus and $\mathrm{CVD}^{7}{ }^{7}$ and chronic disease management incorporating precision medicine will be realised in the near future.

In traditional Korean medicine (KM), individualised disease treatment and health management consider KM types, such as 


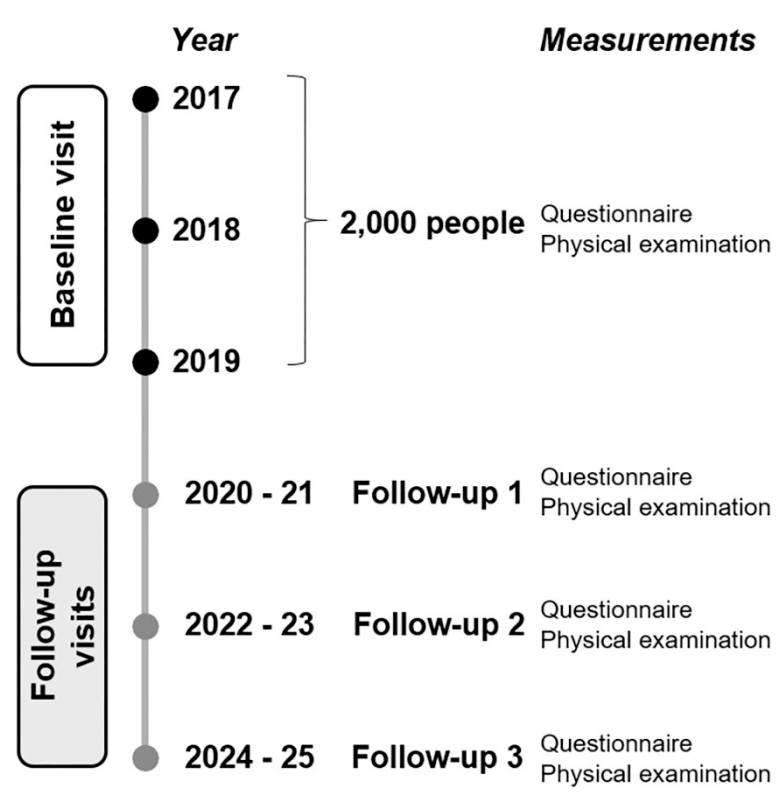

Figure 1 Flow of the Korean Medicine Daejeon Citizen Cohort study.

constitution types and cold-heat patterns. Multiple studies have found that constitution is an independent risk factor for chronic diseases, such as metabolic syndrome, ${ }^{8}$ hypertension, ${ }^{9}{ }^{10}$ diabetes mellitus ${ }^{11}$ and $\mathrm{CVD}^{12}$; and several studies have confirmed the efficacy of several Korean medical treatment techniques in the management of chronic diseases. With constitution and cold-heat found to be heritable by family ${ }^{1314}$ and $\operatorname{twin}^{1516}$ studies, there is active ongoing research on heredity indices, such as whole genome studies.

Here, we describe the design of the Korean Medicine Daejeon Citizen Cohort (KDCC) study, a prospective community-based cohort in Korea. The study has been planned to develop precise health promotion model for the prevention and treatment of chronic diseases, and it considers individual lifestyles and clinical data based on an analysis of individual genomic characteristics with reference to previous cross-sectional studies.

\section{OBJECTIVES}

The aim of the KDCC study is to develop a precise prevention and treatment model for chronic diseases that considers lifestyles and clinical data based on individual KM types as interpreted with the genome. The specific objectives for this cohort are as follows:

1. Establish a sustainable KM cohort.

2. Develop a genome-based diagnostic technology using KM types, such as constitution types and cold-heat patterns.

3. Identify the important factors of lifestyle related to chronic disease and health-related quality of life (HRQOL) according to the KM type.

4. Propose a health promotion model for the prevention and treatment of chronic diseases and for improving the HRQOL that considers KM types and individual traits.

\section{METHODS AND ANALYSIS \\ Study design and setting}

The KDCC is a prospective cohort study based in a region of South Korea. This cohort study is being conducted over a 9-year period between 2017 and 2025, with 2000 adults aged 30-55 years living in Daejeon. Participants will visit the hospital four times for study purposes: for the baseline study (2017-2019) and for three follow-ups every 2 years (2020-2025). The study schedule is summarised in figure 1. Stratified cluster sampling was used to obtain the sample. The entire 30-55 years adult population in Daejeon was set as the population, ${ }^{17}$ which was clustered according to region (five administrative districts called ' $\mathrm{Gu}$ ' based on the address of residence), sex (male or female) and age (30-39 years, 40-49 years and 50-55 years). The sample was extracted based on the target sample size and the probability proportion of the population in each cluster.

This study is conducted in a joint collaboration among the Korea Institute of Oriental Medicine, Gallup Korea and Korean Medicine Hospital of Daejeon University. All information will be acquired by well-trained expert interviewers and researchers. The questionnaire consists of items concerning general characteristics, disease history, lifestyle, HRQOL and KM clinical information. An expert interviewers at Gallup Korea will conduct face-to-face interviews, using a structured questionnaire, to obtain said information. Health examinations include physical examination and laboratory tests, such as anthropometric measurements, cardiovascular function, body composition and blood tests (for details, see tables 1 and 2). All health examinations will be performed at the Korean Medicine Hospital of Daejeon University.

\section{Study population}

Participants were recruited between June 2017 and December 2019. Eligible individuals were identified by key members of researchers, who were trained regarding the inclusion/exclusion criteria and the procedures involved in recruitment. We examined preliminary suitability based on participants' self-reported information. Potential participants visited the hospital to have their suitability further checked by the medical staff through a detailed examination. Medical staff individually interviewed participants for about $20 \mathrm{~min}$ to explain the cohort study and determine participants' suitability.

The inclusion criteria are as follows: (1) men and women aged 30-55 years, (2) residents of Daejeon and (3) individuals who provided informed consent. However, (1) individuals diagnosed with cancer (malignant tumour) or CVD (myocardial infarction, angina, stroke/apoplexy); (2) individuals deemed to have difficulty following study instructions, such as having difficulty completing and understanding the questionnaire and (3) 
Table 1 Health questionnaire and physical examination in the Korean Medicine Daejeon Citizen Cohort study

\begin{tabular}{ll} 
Classification & Measures and instruments \\
\hline General characteristics & (Questionnaire) Sociodemographic information, smoking, drinking \\
Medical history & (Questionnaire) Disease history, family history, menstrual history \\
Lifestyle & $\begin{array}{l}\text { (Questionnaire) Sleep, amount of activity, diet (dietary pattern, nutrition index, food } \\
\text { frequency questionnaire) }\end{array}$ \\
Health-related quality of life & (Questionnaire) Health-related quality of life, psychological stress \\
Korean medical information & $\begin{array}{l}\text { (Questionnaire) Constitution, cold-heat, sub-health status (Mibyeong) } \\
\text { (Measurement) Facial image, body temperature, iris testing }\end{array}$ \\
Anthropometric measurements & (Measurement) Height, weight, waist/hip circumference, grip strength (hand) \\
Cardiovascular function & (Device) Blood pressure/pulse, pulse wave, heart rate variability \\
Body composition & (Device) Body composition analysis \\
\hline
\end{tabular}

individuals determined by the researcher to be inappropriate to participate in this study were excluded.

\section{Baseline and follow-up visits}

This study is currently planned as a 9-year study. Cohort registration and baseline surveys, which began in June 2017, were completed in December 2019. On conclusion of registration, follow-up surveys will be performed three times from 2020 to 2025 in 2-year intervals (first follow-up, 2020-2021; second follow-up, 2022-2023 and third

Table 2 Blood tests and human biological materials in the Korean Medicine Daejeon Citizen Cohort study

\begin{tabular}{|c|c|}
\hline Classification & Measures \\
\hline $\begin{array}{l}\text { Complete blood } \\
\text { count }\end{array}$ & $\begin{array}{l}\text { White blood cells, red blood cells, } \\
\text { haemoglobin, hematocrit, mean } \\
\text { corpuscular volume, mean corpuscular } \\
\text { haemoglobin, mean corpuscular } \\
\text { haemoglobin concentration, red cell } \\
\text { distribution width, platelet count, } \\
\text { Eosinophil count }\end{array}$ \\
\hline Renal panel & Blood urea nitrogen, creatinine \\
\hline $\begin{array}{l}\text { Liver function } \\
\text { test }\end{array}$ & $\begin{array}{l}\text { Total protein, albumin, alkaline } \\
\text { phosphatase, aspartate aminotransferase, } \\
\text { alanine aminotransferase, total bilirubin, } \\
\text { direct bilirubin, r-GTP }\end{array}$ \\
\hline Lipid profile & $\begin{array}{l}\text { Total cholesterol, triglyceride, high density } \\
\text { lipoprotein cholesterol, low density } \\
\text { lipoprotein cholesterol }\end{array}$ \\
\hline $\begin{array}{l}\text { Glucose } \\
\text { metabolism }\end{array}$ & Glucose, HbA1C, insulin \\
\hline Thyroid function & TSH, T4, T3 \\
\hline Inflammation & High sensitivity $\mathrm{C}$ reactive protein, \\
\hline Others & Calcium \\
\hline $\begin{array}{l}\text { Human biological } \\
\text { materials }\end{array}$ & $\begin{array}{l}\text { Blood: DNA, serum, peripheral blood } \\
\text { mononuclear cell }\end{array}$ \\
\hline
\end{tabular}

HbA1C, hemoglobin A1c; r-GTP, gamma-glutamyl transpeptidase; T3, triiodothyronine ; T4, thyroxine; TSH, thyroid-stimulating hormone. follow-up, 2024-2025). During the follow-ups, a questionnaire survey will be administered, and health examinations will be performed including all items collected at baseline (table 3). To minimise follow-up losses, participants will be contacted via phone and mail. If the first phone call attempt fails, additional calls will be made on other days and at other times for up to five times. We plan to update any changes to addresses and contact numbers and to maintain awareness among participants by providing them with study explanations. Further, we plan to send out a study newsletter and thank-you cards periodically.

Table 3 Details and procedures of the baseline study visit and follow-up periods

\begin{tabular}{|c|c|c|c|c|}
\hline \multirow[b]{2}{*}{ Classification } & \multicolumn{4}{|c|}{ Follow-up period } \\
\hline & Baseline & 2 years & 4 years & 6 years \\
\hline $\begin{array}{l}\text { Participant } \\
\text { enrolment }\end{array}$ & $x$ & - & - & - \\
\hline Informed consent & $\mathrm{X}$ & $x$ & $x$ & $x$ \\
\hline $\begin{array}{l}\text { General } \\
\text { characteristics }\end{array}$ & $x$ & $x$ & $x$ & $x$ \\
\hline Medical history & $x$ & $x$ & $x$ & $x$ \\
\hline Lifestyle & $x$ & $x$ & $x$ & $x$ \\
\hline $\begin{array}{l}\text { Health-related } \\
\text { quality of life }\end{array}$ & $x$ & $x$ & $x$ & $x$ \\
\hline $\begin{array}{l}\text { Korean medical } \\
\text { information }\end{array}$ & $x$ & $x$ & $x$ & $x$ \\
\hline $\begin{array}{l}\text { Anthropometric } \\
\text { measurements }\end{array}$ & $x$ & $x$ & $X$ & $x$ \\
\hline $\begin{array}{l}\text { Cardiovascular } \\
\text { function }\end{array}$ & $X$ & $x$ & $X$ & $x$ \\
\hline Body composition & $x$ & $x$ & $x$ & $x$ \\
\hline Blood test & $\mathrm{X}$ & $x$ & $x$ & $x$ \\
\hline $\begin{array}{l}\text { Biological } \\
\text { materials }\end{array}$ & $\mathrm{X}$ & $X$ & $x$ & $X$ \\
\hline
\end{tabular}




\section{Outcome events}

The primary outcome in this study is new onset of CVD, such as myocardial infarction, angina, or stroke, during participation; and the endpoint is either death from CVD or the conclusion of the study period. The secondary outcome is the onset of chronic diseases, such as metabolic syndrome, hypertension, diabetes mellitus, obesity and dyslipidaemia. Finally, we plan to examine the characteristics of risk factors related to changes in subjective health status, such as HRQOL. When the standardised, structured questionnaire identifies a potential outcome event, physicians and appropriate specialists will verify and adjudicate the information.

Within the legally allowed range, the diagnosis of myocardial infarction, angina and stroke will be determined by direct follow-up through participant recontact as well as indirect tracking using medical insurance and official statistical record of death linkage based on International Classification of Disease 10th revision. Furthermore, the secondary outcomes are defined as follows based on the international guidelines for the diagnosis of each disease: metabolic syndrome is defined according to the National Cholesterol Education Program-Adult Treatment Panel III (NCEP-ATP III), ${ }^{18}$ hypertension is defined according to the Joint National Committee, ${ }^{19} 20$ diabetes mellitus is defined according to the American Diabetes Association, ${ }^{21}$ obesity is defined by WHO AsiaPacific Guideline ${ }^{22}$ and Korean Society for the Study of Obesity, ${ }^{23}$ and dyslipidaemia is defined according to the NCEP-ATP III. ${ }^{18}$

\section{Sample size calculations}

To assess the suitability of the suggested sample size $(n=2000)$, we considered various genetic models and disease incidences. This is because identifying significant biomarkers for KM types is one of the important goals of this study. We explored the appropriate sample size according to the Allelic model, dominant model, codominant model and recessive model, which are used in Genome-Wide Association Studies (GWAS) with the incidence of chronic disease set to $20 \%$ and ORs between genotype and disease set to 1.3, 1.5. 2 and 2.5. ${ }^{24}$ Power and alpha were set to $80 \%$ and $5 \%$, respectively, and the ratio of the disease group to normal group was set to $1: 4$, with minor allele frequency of $5 \%$ and complete linkage disequilibrium (D') equal to one. We confirmed that about 300-500 patients are needed for the genotype OR for chronic disease to increase by two or more. Thus, based on the incidence of disease and statistical power in this study, we set the sample size to 2000 .

\section{Measurement and instruments}

\section{General information and medical history}

As general attributes, sociodemographic characteristics such as sex, age, occupation, education, marital status, number of hours worked and family income are surveyed.

For smoking, the following questions are asked: 'Have you smoked more than 100 cigarettes in your entire life?' and 'Do you currently smoke?' Participants are then classified according to their smoking status (ie, currently smoking, smoked in the past and non-smoker), and the age they first started smoking and the period of smoking are further investigated. In addition, for drinking, the following questions are asked: 'Have you consumed alcohol in the past?' and 'Do you currently drink alcohol?' Participants are then classified according to their drinking status (ie, currently drinking, drank in the past and non-drinker) and the total period of drinking as well as average frequency of drinking for the past year and the amount of drinking per incidence according to the type of a drink (unrefined rice wine, refined rice wine, wine, soju, beer, liquor, etc) are further investigated.

For medical history, about 40 diseases are listed, and the status of each of these diseases is investigated based on a hospital diagnosis, along with family history of the diseases. For female participants, information about menstrual status is collected.

\section{KM clinical information}

To classify the KM types of individuals, constitution types and cold-heat patterns are examined. Constitution types and cold-heat patterns can be assessed using a previously validated questionnaire with established classification criteria and can be used to classify individual KM types. To assess constitution, the simplified Korea Sasang Constitution Diagnostic Questionnaire comprising 15 questions on body type (one question on physical trait, six questions on personality traits, and eight questions on physiological symptoms) are administered. ${ }^{25} 26$ Cold-heat patterns are assessed using two types of tools. First, the Cold-Heat Pattern Identification, which consists of eight items for cold pattern and seven items for heat pattern, is used to assess daily symptoms. ${ }^{27}$ The Cronbach's alpha coefficients representing internal consistency are 0.79 (cold) and 0.83 (heat). ${ }^{27} 28$ Then, the seven-item Short Form Cold/Heat Questionnaire (SF-CHQ), which can identify the traits of specific parts of the body, such as the hands, feet, and abdomen and cold-heat sensitivity, is used. The correlation coefficient for test-retest reliability for the SF-CHQ was 0.609 , and classification accuracy against expert classification was $74.5 \%{ }^{29}$

Subhealth status is assessed using a Mibyeong questionnaire. The Mibyeong questionnaire evaluates an individual's subhealth status based on subjective symptoms, and it consists of 21 items regarding the persistence of, discomfort from, and recovery from seven conditions (fatigue, pain, sleep, indigestion, anxiety, depression and anger) after rest. Based on the total score on the Mibyeong questionnaire, individuals are classified into healthy, mild Mibyeong and severe Mibyeong. ${ }^{30} 31$

The colour and texture of the face are important indicators of health in KM. Thus, we collected facial photographs of the cohort (using a Nikon DSLR D5100 with $50 \mathrm{~mm}$ lens), and per the standard operating procedure, distance, lighting and posture were controlled during the photographing. ${ }^{32}$ The front and side of the faces 
are photographed using a facial imaging programme with participants going without make-up as much as possible. Further, as one of the methods to assess coldheat patterns, body surface temperature (using an IRISXP, Medicore, Seoul, Korea) and iris images (using a Camscope Pro, Sometech, Seoul, Korea) are taken.

\section{Lifestyle}

In this study, we observe sleep, physical activity and diet pattern as lifestyle variables. The components of sleep status, sleep duration and sleep quality, is investigated using the Korean Pittsburgh Sleep Quality Index (PSQI). ${ }^{33}{ }^{34}$ The PSQI measures sleep quality and disturbances during the previous month using 19 items that assess a broad range of domains related to sleep quality. ${ }^{34}$ These items are combined to form seven component scores: subjective sleep quality, sleep latency, sleep duration, habitual sleep efficiency, sleep disturbances, use of sleeping medication and daytime dysfunction. Each component score has a range of $0-3$, and the sum of the seven components yields a global score ranging from 0 to 21 , with the higher scores indicating more severe sleep complaints.

Physical activity is assessed using the Korean Global Physical Activity Questionnaire (GPAQ) developed by WHO. ${ }^{35}{ }^{36}$ The GPAQ can assess the amount of physical activity by domain, such as work-related activity, activity when moving to another location and leisure activity. It also measures the intensity levels of physical activity by converting it to a metabolic equivalent task-commonly used in the analysis of physical activity.

Diet is assessed using three questionnaires: dietary behaviours, nutrition quotient (NQ) and the Food Frequency Questionnaire (FFQ). For dietary behaviours, meal frequency (average frequency, specific frequency of breakfast, lunch and dinner), meal regularity, meal speed and frequency of overeating/snacking/dining out are surveyed. NQ a questionnaire developed by the Korea Nutrition and Health Society, consists of items about intake of 12 major food groups and nine diet behaviours. The NQ questionnaire can be used to assess adults' meal quality and nutrition intake status, and the questionnaire and scoring method have been validated. ${ }^{37}$ The FFQ assesses the average frequency of food intake in the past year, and we will use a modified version based on the items in the 2011 Korea Health and Nutrition Examination Survey. ${ }^{38} 39$

\section{HRQOL and stress}

HRQOL is assessed using the SF-12 version 2 and EuroQoL 5-dimension (EQ-5D). The SF-12 is a 12-item scale that assesses overall HRQOL based on physical and mental health status. ${ }^{40}$ It contains eight categories (physical functioning, physical role restriction, pain, general health, mental health, emotional role restriction, social functioning and vitality), and their scores are used to calculate the physical component summary-12 (PCS) and mental component summary-12 (MCS) scores. The
PCS and MCS are scored from 0 to 100 per a norm-based scoring algorithm, and a higher score indicates a better HRQOL. The EQ-5D was developed by the EQ Group as a generic measure of HRQOL. ${ }^{41} 42$ This tool assesses health status in five dimensions (mobility, self-care, usual activities, pain/discomfort and anxiety/depression) with three levels (no problems, some or moderate problems and extreme problems), which was called EQ-5D index, and $100 \mathrm{~mm}$ Visual Analogue Scales (EQ-5D VAS) with vertical lines. EQ-5D index provides an estimate of the health summary score by applying a formula that attaches specific values to each level in each dimension. EQ-5D index scores are calculated using a time trade-off valuation set that pertains to the Korean population.$^{30}$ The total score ranges from 0 to 1 , with a lower score indicating a poorer health status. EQ-5D VAS scores range from worst (0) to optimum health (100). Both the SF-12 and EQ-5D assess the overall HRQOL, but a comparison of the two scales shows that EQ-5D can be used for economic assessment (quality-adjusted life year) but has a ceiling effect. Thus, the two questionnaires are combined for various purposes, such as health status evaluation. ${ }^{43}$

Psychological stress is assessed using the 18-item Psychosocial Well-being Index-SF (PWI-SF). ${ }^{44}$ Each item on PWI-SF is rated on a four-point scale from 0 (always true) to 3 (never true), and the total score ranges from 0 to 54 , with a higher score indicating a higher level of stress.

\section{Anthropometric measurements}

Anthropometry is taken by trained personnel with participants wearing only light underwear. Body height (in $\mathrm{cm}$ ) and weight (in $\mathrm{kg}$ ) is measured with the measuring station BSM370 and Inbody 770 (Biospace), and waist and hip circumference (both in $\mathrm{cm}$ ) are taken with a tape measure (Hoechstmass-Rollfix, Germany) according to WHO guidelines. For grip strength, a hand dynamometer (TKK 5401, Takei Scientific Instruments, Niigata, Japan) is used to measure maximum strength in the right and left hands. Body circumferences and grip strength are each measured twice.

\section{Cardiovascular function assessment}

For cardiovascular function assessment, blood pressure, pulse rate, pulse wave and heart rate variability are measured with the participant sitting down and after $5-10 \mathrm{~min}$ of rest. Blood pressure and pulse rate are measured twice using an automatic blood pressure cuff (FT-500R PLUS, Jawon medica, Korea). The second measurement is taken 5 min after the first measurement in the same position, and if there is a significant difference between the first and second measurements (eg, $\geq 5-10 \mathrm{~mm} \mathrm{Hg}$ ), a third measurement is taken. Pulse wave is measured with a DMP-1000 (Daeyomedi, Korea) using the tonometry method, where pressure is measured while compressing the left radial artery (vascular area). Pulse waves show the features of a pulse, circulation resistance and vascular anomaly. Heart rate variability is measured with the SA6000P (Medicore) for 5 min after attaching 
ECG sensors on the right and left wrists and left ankle. The level of autonomic nervous system function is assessed using low frequency and high frequency values computed based on an algorithm.

\section{Body composition}

We assess body composition by measuring bioimpedence with the InBody 770 (Biospace) and along six frequencies $(1 \mathrm{kHz}, 5 \mathrm{kHz}, 50 \mathrm{kHz}, 250 \mathrm{kHz}, 500 \mathrm{kHz}$ and $1 \mathrm{MHz})$ at five locations: right arm, left arm, trunk, right leg and left leg. The manufacturer recommends that participants stand barefoot on the electrodes and while holding the hand electrodes wearing light clothing and no accessories. Arms and legs should not contact any other parts of the body and should instead be spread. Bioimpedence analysis allows non-invasive, easy and an inexpensive assessment of body composition and is applicable to a wide range of subjects.

\section{Blood test and human resources}

A venous blood sample (about $22.5 \mathrm{~mL}$ ) is taken in the morning following overnight fasting. Thirty minutes after collection, the sample is centrifuged for $10 \mathrm{~min}$ at $3450 \mathrm{rpm}$, and all samples are transported to the Seoul Clinical Laboratories (Seoul, Korea) within 24 hours. As shown in table 2, the blood test includes complete blood count, kidney and liver function, lipid profile, glucose metabolism, thyroid function and inflammatory marker. Further, for a genomic study, DNA, serum and peripheral blood mononuclear cell are collected and are stored at $-80^{\circ} \mathrm{C}$ per the standard procedure until use.

\section{Data analysis}

The detailed data analysis plan for genomic research and clinical epidemiology research in this study is as follows. For genomic research, heritability in KM types including constitution types and cold-heat pattern has been reported in previous family ${ }^{1314}$ and $\operatorname{twin}^{1516}$ studies. In this study, using the GWAS-convolutional neural network, an improvement on the existing GWAS analysis method, ${ }^{45} \mathrm{KM}$ types of the cohort participants will be predicted. For the clinical epidemiology analysis, using previous studies on lifestyle factors such as sleep, physical activity, eating habit, smoking and drinking, ${ }^{46} 47$ we will divide responses as healthy and unhealthy lifestyle based on a clustering phenomenon, and the factors influencing CVD, metabolic syndrome and relevant diseases will be analysed according to KM types deduced from genomic research.

In addition, we will perform a variety of analyses conducted in cohort studies. To estimate cumulative risk of primary and secondary outcomes, the study will employ Kaplan-Meier analysis. Adjustments for covariates will be made prior to the follow-up period where Cox proportional hazard models will estimate the risk of experiencing any of the primary or secondary outcomes. We will also conduct cross-sectional analysis. Categorical variables will be compared using $\mathrm{X}^{2}$ tests, while continuous variables will be compared using the Student's t-test and the analysis of variance. In case of skewed distributions which cannot be normalised corresponding, nonparametric tests will be used.

\section{Data management and protection}

All data collected in this cohort study are managed and stored by the Korean Medicine Data Center (KDC) of the Korean Institute of Oriental Medicine. All data are electronically managed using the KDC electronic data capture (EDC) system, a secure, web-based application that allows for data instruments to be created, data quality to be monitored and data analysis to be completed (https://ecrf.kiom.re.kr/). The KDC EDC system has restricted physical access; data are stored under coded filenames, and the network has secure password access restricted to a limited number of people. All researchers will be trained in data entry by the KDC administrator and must demonstrate proficiency with mock patients and data entry before being granted access to data collection forms. All records containing personal health information will be kept confidential and, to the extent permitted by the applicable laws and/or regulations, will not be made publicly available. Deidentified data may be used for future analysis and publication.

\section{Ethics and dissemination}

All participants will provide written informed consent and consent to the collection of human biological materials. All participants are informed that they can withdraw their consent to participate at any point. There are no anticipated physical, social, legal or economic risks associated with the study. There is minimal risk of breach of confidentiality. No vulnerable populations are specifically targeted in this study. Results from this study will be disseminated at regional and international conferences and in peer-reviewed journals.

\section{Researchers' training and cooperation}

The primary investigator has extensive training and experience in clinical research and relevant bioethics. The researchers will include a team of primary investigator that have extensive qualifications and expertise to lead the study. All study staff, including interviewers and medical staff who collect the data, are trained and routinely re-educated about the ethical conduct of human subject research. Further, researchers from various disciplines will cooperate to develop a cohort study protocol, manage and conduct the research, and analyse clinical and genomic data. The researchers in this study hold regular meetings to discuss the study design, progress and clinical issues; and they also participate in theoretical and practical training sessions. All research processes including participant recruitment, obtaining consent and data collection follow the standard operating procedure.

\section{Patient and public involvement}

There was no patient or public involvement in this study as it describes a study protocol. 


\section{DISCUSSION}

This protocol describes the design and method of the KDCC study in detail. The KDCC study aims to assess genomic-based KM types, lifestyle and chronic disease status in middle-aged residents of Daejeon, Korea, through a series of long-term follow-up examinations. In this study, we focus on discovering biomarkers that define the $\mathrm{KM}$ types of an individual based on the genome in the short term. In the long term, we aim to examine how the associations between chronic disease and HRQOL vary across different patterns of the KM types. Ultimately, this study will evaluate the impact of the varying lifestyle according to KM types on chronic disease and health status, and will discover a genetic marker for KM types, such as constitution types and cold-heat patterns, based on genomic analysis to develop a technique for predicting them.

This study has several strengths. First, it is a long-term cohort study. Thus, the study will gather important data to assess the causal relationships among KM types, lifestyle and chronic disease. Second, it is the first KM-centred prospective cohort study. Hence, various clinical data and genomic data obtained in this study could be used as evidence to support the clinical value of traditional Korean precision medicine. Third, this study sheds light on genomic-based KM types. Whereas prior research relied on expert-dependent studies or questionnairebased studies, this study will perform a genomic analysis and thus can produce more objective results than prior studies that used genomic analysis, as well as presenting key results to understand the biological features of KM types. Fourth, the questionnaires are administered via a face-to-face interview; thus, it will produce more accurate information than self-reported data by using standardised methods. Finally, the KDCC is implemented by a multidisciplinary research team comprising experts in traditional KM, genetics, statistics and epidemiology. The combination of diverse perspectives and expertise among team will enable novel and original research into health management using traditional Korean precision medicine. Despite these strengths, the greatest limitations of this study are the possibility of selection bias owing to voluntary participation and the limitation of the generalisability of the findings to the Korean adult population, as the participants were sampled from one region.

The KDCC study is expected to present key evidence and model for the development of traditional Korean precision medicine by examining the impact of the interaction among KM types, lifestyle factors and various clinical information on chronic disease and health status. Moreover, our data could be used as foundational data for further research. Currently, we plan to conduct this study on 2000 people for a minimum of 9 years; however, we hope to increase the sample size and extend the follow-up period depending on the availability of resources.

Acknowledgements This study was supported by the Korea Institute of Oriental Medicine (no. KSN2021120).
Contributors YB, B-NS and SL made substantial contributions to the conception and design of this protocol. YB, B-NS and KJ critically read and revised this manuscript. SL and HY approved this final version for submission. All authors agree to be accountable for the future integrity of this study.

Funding This study was supported by the 'Development of Korean Medicine Original Technology for Preventive Treatment based on Integrative Big Data' grant from the Korea Institute of Oriental Medicine (no. KSN2021120).

Competing interests None declared.

Patient and public involvement Patients and/or the public were not involved in the design, or conduct, or reporting, or dissemination plans of this research.

Patient consent for publication Not required.

Ethics approval All aspects of this study, including written informed consent, data collection and photo acquisition, were approved by the Institutional Review Board of the Dunsan Korean Medicine Hospital of Daejeon University (no. DJDSKH17-BM-12) and the Korea Institute of Oriental Medicine Institutional Review Board (no. I-1703/002-002).

Provenance and peer review Not commissioned; externally peer reviewed.

Open access This is an open access article distributed in accordance with the Creative Commons Attribution Non Commercial (CC BY-NC 4.0) license, which permits others to distribute, remix, adapt, build upon this work non-commercially, and license their derivative works on different terms, provided the original work is properly cited, appropriate credit is given, any changes made indicated, and the use is non-commercial. See: http://creativecommons.org/licenses/by-nc/4.0/.

ORCID iD

Younghwa Baek http://orcid.org/0000-0002-1827-1701

\section{REFERENCES}

1 World Health Organization. Global status report on noncommunicable diseases, 2014. Available: https://www.who.int/ ncds/introduction/en/ [Accessed 9, Aug 2019].

2 World Health Organization. Global health estimates 2016: deaths by cause, age, sex, by Countryand by region, 2000-2016. Geneva: World Health Organization, 2018.

3 Cao B, Bray F, llbawi A, et al. Effect on longevity of one-third reduction in premature mortality from non-communicable diseases by 2030: a global analysis of the sustainable development goal health target. Lancet Glob Health 2018;6:e1288-96.

4 World Health Organization. Cardiovascular diseases. Available: https://www.who.int/health-topics/cardiovascular-diseases/ [Accessed 7 August 2019].

5 Piepoli MF, Hoes AW, Agewall S, et al. 2016 European Guidelines on cardiovascular disease prevention in clinical practice: The Sixth Joint Task Force of the European Society of Cardiology and Other Societies on Cardiovascular Disease Prevention in Clinical Practice (constituted by representatives of 10 societies and by invited experts) Developed with the special contribution of the European Association for Cardiovascular Prevention \& Rehabilitation (EACPR). Eur Heart $J$ 2016;37:2315-81.

6 Janssen V, De Gucht V, Dusseldorp E, et al. Lifestyle modification programmes for patients with coronary heart disease: a systematic review and meta-analysis of randomized controlled trials. Eur J Prev Cardiol 2013;20:620-40.

7 Collins FS, Varmus $\mathrm{H}$. A new initiative on precision medicine. N Engl J Med 2015;372:793-5.

8 Song KH, Yu S-G, Kim JY. Prevalence of metabolic syndrome according to Sasang constitutional medicine in Korean subjects. Evid Based Complement Alternat Med 2012;2012:1-8.

9 Lee J, Lee J, Lee E, et al. The Sasang constitutional types can act as a risk factor for hypertension. Clin Exp Hypertens 2011;33:525-32.

10 Lee SK, Yoon DW, Lee SW, et al. Association of Sasang constitutional types with incident hypertension: a 12-year follow-up study. J Altern Complement Med 2016;22:706-12.

11 Lee T-G, Koh B, Lee S. Sasang constitution as a risk factor for diabetes mellitus: a cross-sectional study. Evid Based Complement Alternat Med 2009;6 Suppl 1:99-103.

12 Cho NH, Kim JY, Kim SS, et al. The relationship of metabolic syndrome and constitutional medicine for the prediction of cardiovascular disease. Diabetes Metab Syndr 2013;7:226-32.

13 Won $\mathrm{H}-\mathrm{H}$, Lee S, Jang E, et al. A genome-wide scan for the Sasang constitution in a Korean family suggests significant linkage at 
chromosomes 8q11.22-23 and 11q22.1-3. J Altern Complement Med 2009;15:765-9.

14 Lee M-K, Jang E-S, Sohn H-Y, et al. Investigation of genetic evidence for Sasang constitution types in South Korea. Genomics Inform 2009;7:107-10.

15 Hur Y-M, Lee S, Jin H-J. Genetic and environmental overlaps among Sasang constitution types: a multivariate twin study. Twin Res Hum Genet 2018;21:518-26.

16 Hur Y-M, Yu H, Jin H-J, et al. Heritability of cold and heat patterns: a twin study. Twin Res Hum Genet 2018;21:227-32.

17 Daejeon Metropolitan City. January 2017 resident registration population in Daejeon: Daejeon, 2017. Available: https://www. daejeon.go.kr/sta/StaStatisticsFldView.do;jsessionid=6K4htwr3Jrvj 71szCVaoa7T2QtOzKOcYhgk1yVC80iqBUkmlgePBQxBG5qU08gOr. WEB1_servlet_engine3?ntatcSeq=1081285802\&menuSeq= $180 \&$ colmn1Cont $=$ C0201\&colmn2Cont $=$ C020101\&pagelndex $=1$ [Accessed 2 Feb 2017].

18 National Cholesterol Education Program (NCEP) Expert Panel on Detection, Evaluation, and Treatment of High Blood Cholesterol in Adults (Adult Treatment Panel III). Third report of the National cholesterol education program (NCEP) expert panel on detection, evaluation, and treatment of high blood cholesterol in adults (adult treatment panel III) final report. Circulation 2002;106:3143.

19 Chobanian AV, Bakris GL, Black HR, et al. The seventh report of the joint National Committee on prevention, detection, evaluation, and treatment of high blood pressure: the JNC 7 report. JAMA 2003;289:2560-71.

20 James PA, Oparil S, Carter BL, et al. 2014 evidence-based guideline for the management of high blood pressure in adults: report from the panel members appointed to the eighth joint National Committee (JNC 8). JAMA 2014;311:507-20.

21 American Diabetes Association. Diagnosis and classification of diabetes mellitus. Diabetes Care 2014;37 Suppl 1:S81-90.

22 World Health Organization. The Asia-Pacific perspective: redefining obesity and its treatment. Sydney: Health Communications Australia, 2000.

23 Lee SY, Park HS, Kim DJ, et al. Appropriate waist circumference cutoff points for central obesity in Korean adults. Diabetes Res Clin Pract 2007;75:72-80.

24 Hong EP, Park JW. Sample size and statistical power calculation in genetic association studies. Genomics Inform 2012;10:117.

25 Baek Y-H, Jang E-S, Park K-H, et al. Development and validation of brief KS-15 (Korea Sasang constitutional diagnostic questionnaire) based on body shape, temperament and symptoms. Journal of Sasang Constitutional Medicine 2015;27:211-21.

26 Kim Y, Jang E. Test-Retest Reliability of Brief KS-15 - Korean Sasang Constitutional Diagnostic Questionnaire -. Kjopp 2016;30:177-83.

27 Yeo M, Park K, Bae K, et al. Development on the questionnaire of Cold-Heat pattern identification based on usual symptoms for health promotion - focused on reliability study. Kjopp 2016;30:116-23.

28 Bae K-H, Yoon Y, Yeo M, et al. Development on the Questionnaire of Cold-Heat Pattern Identification Based on Usual Symptoms for Health Promotion - Focused on Agreement Study. J Soc Prev Korean Med 2016;20:17-26.

29 Yoon Y, Kim H, Lee Y, et al. Developing an optimized cold/heat questionnaire. Integr Med Res 2015;4:225-30.
30 Jang E, Yoon J-H, Lee Y. The evaluation of reliability and validity for Mibyeong questionnaire. J Korea Instit Orient Med Diagn 2017;21:13-25.

31 Lee Y, Baek Y, Park K, et al. Development and validation of an instrument to measure the health status of healthy but unsatisfied people: Mibyeong index. J Soc Prev Korean Med 2016;20:45-53.

32 Pham DD, Do J-H, Ku B, et al. Body mass index and facial cues in sasang typology for young and elderly persons. Evid Based Complement Alternat Med 2011;2011:1-9.

33 Sohn SI, Kim DH, Lee MY, et al. The reliability and validity of the Korean version of the Pittsburgh sleep quality index. Sleep Breath 2012;16:803-12.

34 Buysse DJ, Reynolds CF, Monk TH, et al. The Pittsburgh sleep quality index: a new instrument for psychiatric practice and research. Psychiatry Res 1989;28:193-213.

35 Armstrong T, Bull F. Development of the world Health organization global physical activity questionnaire (GPAQ). J Public Health 2006;14:66-70.

36 Jeon JY. Development of the Korean version of global physical activity questionnaire and assessment of reliability and validity. Osong. Korea: Korea Centers for Disease Control and Prevention, 2013: 1-105.

37 Lee J-S, Kim H-Y, Hwang J-Y, et al. Development of nutrition quotient for Korean adults: item selection and validation of factor structure. J Nutr Health 2018;51:340-56.

38 Ministry of Health and Welfare, Korea Centers for Disease Control and Prevention. Korea health statistics 2011: Korea National health and nutrition examination survey (KNHANES V-2. Cheongwon: Korea Centers for Disease Control and Prevention, 2012.

39 Lee S, Kim Y, Seo S, et al. A study on dietary habits and food intakes in adults aged 50 or older according to depression status. J Nutr Health 2014;47:67-76.

40 Ware J, Kosinski M, Keller SD. A 12-Item short-form health survey: construction of scales and preliminary tests of reliability and validity. Med Care 1996;34:220-33.

41 Brooks R, Group E. EuroQol: the current state of play. Health Policy 1996;37:53-72.

42 Lee Y-K, Nam H-S, Chuang L-H, et al. South Korean time trade-off values for EQ-5D health states: modeling with observed values for 101 health states. Value Health 2009;12:1187-93.

43 Johnson JA, Pickard AS, ASJMc P. Comparison of the EQ-5D and SF-12 health surveys in a general population survey in Alberta, Canada. Med Care 2000;38:115-21.

44 Chang S. Standardization of collection and measurement for Heath data. Seoul: Kyechukmunhwasa, 2000.

45 Lee T, Sung MK, Lee S, et al. Convolutional neural network model to predict causal risk factors that share complex regulatory features. Nucleic Acids Res 2019;47:e146-e46.

46 Li Y, Pan A, Wang DD, et al. Impact of healthy lifestyle factors on life expectancies in the US population. Circulation 2018;138:345-55.

47 Li Y, Schoufour J, Wang DD, et al. Healthy lifestyle and life expectancy free of cancer, cardiovascular disease, and type 2 diabetes: prospective cohort study. BMJ 2020;368:16669. 